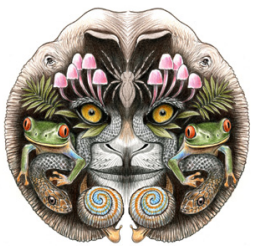

ISSN

Online 0974-7907

Print 0974-7893

\title{
SPECIES DIVERSITY AND CONSERVATION OF AVIFAUNA IN THREE DIFFERENT HABITAT TYPES WITHIN THE MIHINTALE SANCTUARY, SRI LANKA
}

OPEN ACCESS

\section{Chathurabhani Wimalasekara ${ }^{1} \&$ Sriyani Wickramasinghe ${ }^{2}$}

${ }^{1,2}$ Department of Biological Science, Faculty of Applied Sciences, Rajarata University of Sri Lanka, Mihintale, Sri Lanka ${ }^{1}$ sriwick@gmail.com (corresponding author), ${ }^{2}$ c.wimalasekara@yahoo.com

Abstract: The present study was carried out in three different habitat types within the Mihintale Sanctuary, namely aquatic, undisturbed forest and disturbed habitat from November 2008 to May 2009. The main objective of the study was to determine the species diversity and identify the threats to the birds. Line transect, point counts and opportunistic survey methods were conducted daily both in the morning and evenings. A total of 130 species of birds belonging to 47 families including 111 breeding residents, 19 winter visitors, four endemic species and four species that are being proposed as endemic species were recorded. It also included 10 rare species, one very rare winter visitor, Zoothera citrina, six nationally threatened and one globally threatened species, Pelecanus philippensis. The number of bird species that were observed in the aquatic habitat, forested habitat and the disturbed habitat were 93,40 and 76 respectively. The results indicate that diversity of birds was significantly higher $(p<0.05)$ in the aquatic habitat. It was revealed that the aquatic habitat provides more suitable niches and food resources for a wide variety of birds. Therefore, species diversity near aquatic habitat was higher compared to that of the forest. Fragmentation of forest into small patches, hunting birds for food and presence of natural predators are the major threats to the avifauna in the sanctuary. Thus, strategies should be developed to conserve the avifaunal diversity in the forest patches. The sanctuary supports different types of native bird species of Sri Lanka and is identified as one of the IBAs in Sri Lanka, it therefore plays an important role in the conservation of birds in Sri Lanka.

Keywords: Avifauna, conservation, diversity, endemic, fragmentation, Mihintale Sanctuary.
Sri Lanka's avifauna is one of the richest in the whole of Asia (Kotagama \& Wijayasingha 1998). About 482 bird species were recorded of which 220 breeding residents and 26 species are endemic to the country (Kotagama et al. 2006). Sri Lanka was identified as an Endemic Bird Area (EBA 124) in 1998 (BirdLife International 2008).

Though avifaunal studies were carried out both in dry (most of the southeastern, eastern, and northern parts of the country) and wet (the mountains and the southwestern part of the country) climatic zones of Sri Lanka, majority of the previous avifaunal studies were conducted in the wet zone. There is very little information about the avifauna of the sanctuaries in the dry zone. Mihintale Sanctuary is one of the important sanctuaries located in the dry zone with very little information on its avifauna. The present study aims to identify the species diversity and conservation status of bird species in three different habitat types namely aquatic, undisturbed forest and disturbed areas within the Mihintale Sanctuary.

\section{Materials and Methods}

The Mihintale Sanctuary (as gazetted on 27/05/1938

in the Sri Lanka Government Gazette No.8370

DOI: http://dx.doi.org/10.11609/JoTT.o3119.5718-25 | ZooBank: urn:Isid:zoobank.org:pub:97A2F141-73DF-4937-9385-03C525605D80

Manuscript details: Ms \# 03119 | Received 11 March 2012 | Final received 09 May 2014 | Finally accepted 12 May 2014

Citation: Wimalasekara, C. \& S. Wickramasinghe (2014). Species diversity and conservation of avifauna in three different habitat types within the Mihintale Sanctuary, Sri Lank. Journal of Threatened Taxa 6(5): 5718-5725; http://dx.doi.org/10.11609/JoTT.03119.5718-25

Copyright: (c) Wimalasekara \& Wickramasinghe 2014. Creative Commons Attribution 4.0 International License. JoTT allows unrestricted use of this article in any medium, reproduction and distribution by providing adequate credit to the authors and the source of publication.

Funding: Faculty of Applied Sciences, Rajarata University of Sri Lanka.

Competing Interest: The authors declare no competing interests.

Acknowledgements: We thank the Department of Biological Sciences, Faculty of Applied Sciences, Rajarata University of Sri Lanka for financial supporting this research. We wish to thank Department of Wildlife Conservation and Archeology for granting permission to conduct the research within the sanctuary. We also extend our sincere gratitude to Prof. S.W. Kotagama for his guidance during the research. 
(Department of Government Printing 1938)), is situated in Kanadara Korale of Nuwaragam Palatha in the Anuradhapura District of the North-Central Province and encompasses an area of 2,470acres (999.6ha). There are no proper demarcated boundaries for the sanctuary. It receives an annual rainfall of 1,000-1,500 mm/year from the north-east monsoon and intermonsoons (March-April and September-October). The main source of livelihood of the residents is Chena cultivation (slash and burn) (Divisional Secretary 2007).

Mihintale Sanctuary comprises mainly of undisturbed areas of semi deciduous forest type. Scrublands, water-edge habitats, highly degraded tertiary forests, and vegetation in archaeological sites are also found. Surveys to study the avifauna in three different study sites namely, aquatic habitat and surroundings near 'Kudakirindegama Wewa' (KK) (about 6ha) $\left(8^{\circ} 20^{\prime} 36.518^{\prime \prime} \mathrm{N} \& 80^{\circ} 31^{\prime} 34.034^{\prime \prime} \mathrm{E}\right)$, Kaludiyapokuna forested area (KP) (about 05ha) $\left(8^{\circ} 20^{\prime} 51.752^{\prime \prime} \mathrm{N}\right.$ \& $80^{\circ} 30^{\prime} 27.498^{\prime \prime} \mathrm{E}$ ) and cleared up forest areas of Mihintale Hill (MH) (about 05ha) (8021'13.893”N \& $80^{\circ} 30^{\prime} 34.711^{\prime \prime} \mathrm{E}$ ) (Fig. 1) were taken up. The study was conducted from $0600-0800 \mathrm{hr}$ and $1600-1830 \mathrm{hr}$ in the morning and in the evening, respectively. Line transect method $(200 \times 50 \mathrm{~m})$ was used for sampling terrestrial habitats while point counts were used for sampling aquatic habitats (Bibby et al. 1993). Four fixed points were selected in aquatic habitats and 20-30 minutes were spent during both the point and the transect count methods. The opportunistic observation method was used since some bird species in the sanctuary could not be observed along the line transects or points. A pair of
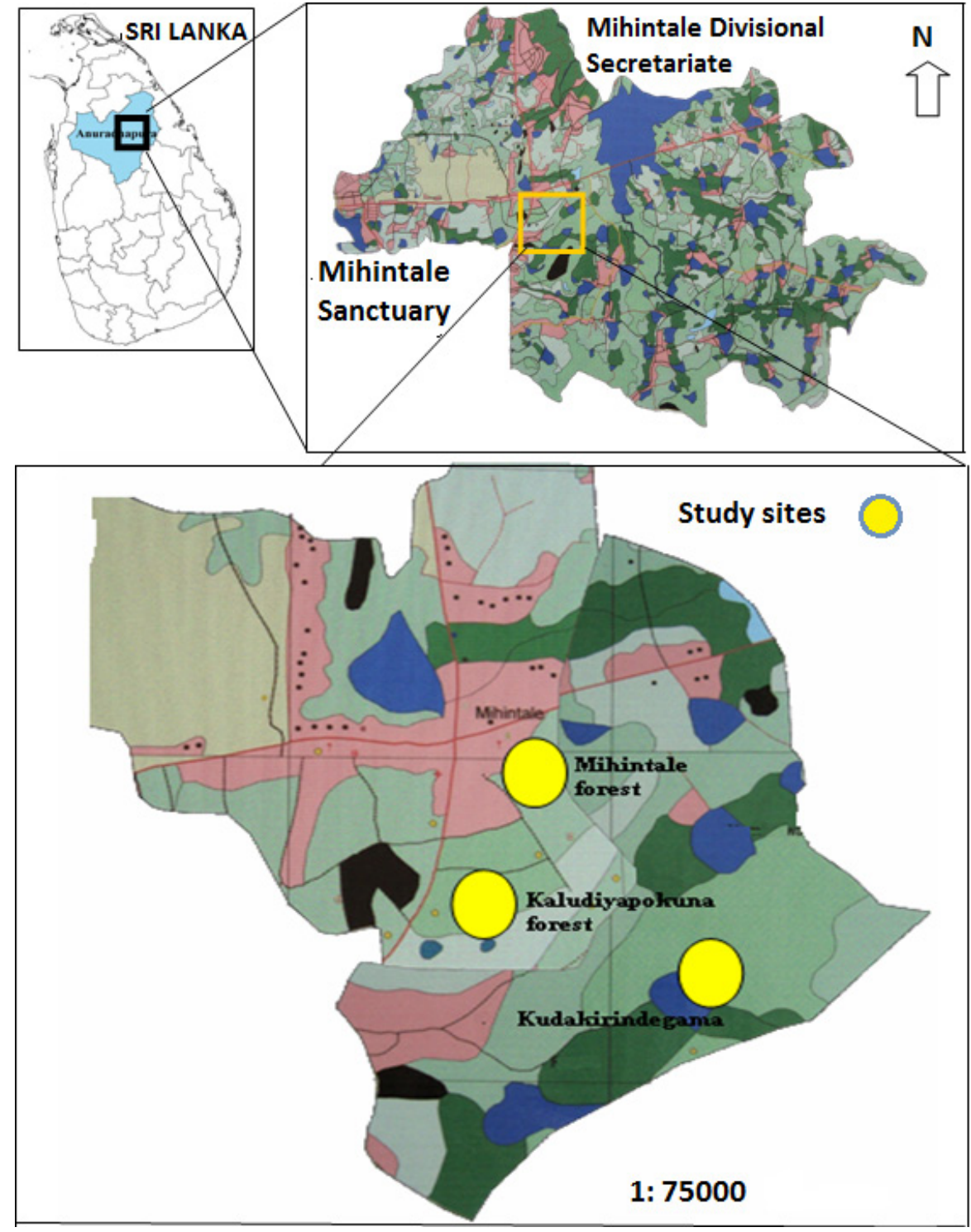

Figure 1. Sampling sites of selected habitats within the Mihintale Sanctuary 
binoculars (Bushnell 13-1056C) was used to identify the birds at a distance and a tally counter was used to count the number of birds sighted. Species identification and nomenclature was based on Kotagama \& Fernando (1994) and Harrison (1999).

Shannon-Wiener Diversity Index was calculated using Microsoft Excel in order to determine the species diversity. Two-way ANOVA was done to compare the avifaunal samples of each habitat using statistical software (MINITAB version 14).

\section{Results}

A total of 130 birds belonging to 47 families were recorded during the seven-month period from November 2008 to May 2009. This included 111 breeding residents, 19 winter visitors, four endemic species and four species that are proposed as endemic birds to Sri Lanka (Kotagama \& Ratnavira 2010). Out of 130 birds, 58 species are very common (100\% occurrence) and 61 are common, including 10 rare species, one very rare winter visitor, Zoothera citrina (Orange-Headed Ground Thrush), one globally threatened species, Pelecanus philippensis (Spot-billed Pelican) and six nationally threatened species (Table 1) (Images 1-4). The total number of bird species observed at KK, KP and $\mathrm{MH}$ were 93, 40 and 76, respectively. The proportions of endemic species recorded as a percentage of the total number of endemic bird species in Sri Lanka were as follows: KP $11.54 \%$ (three species), KK $11.54 \%$ (three species) and $\mathrm{MH} 7.7 \%$ (two species). Shannon Diversity Index $\left(\mathrm{H}^{\prime}\right)$ for $\mathrm{KK}, \mathrm{KP}$ and $\mathrm{MH}$ were 3.83, 3.32 and 3.79, respectively.

The species diversity was significantly high in KK $(P=0.000)$ than the other sites. When compared to the individual numbers in $\mathrm{KK}$ habitat it was significantly higher $(\mathrm{P}=0.000)$ than the other sites (Table 2).

The species composition in the three study areas is as shown in Fig. 2. Thirty-six (27.7\%) bird species observed were confined to Kudakirindegama aquatic area (KK), 21 species $(16.15 \%)$ to Mihintale disturbed area $(\mathrm{MH})$ and 12 species (9.23\%) were exclusive to Kaludiyapokuna forested area (KP). Thirty-three species were recorded only at $\mathrm{KK}$ and $\mathrm{MH}$, four species were recorded only at $\mathrm{KP}$ and $\mathrm{MH}$, six species were recorded only at KK and $\mathrm{KP}$ while $18(13.85 \%)$ species were common to all the three habitats.

Copsychus malabaricus, Nectarinia zeylonica, Oriolus xanthornus are the dominant birds observed at KP. Ardeola grayii, Phalacrocorax fuscicollis, Psittacula kramerii are some of the commonest birds observed at KK. Pycnonotus cafer, Corvus macrorhynchos and Streptopelia chinensis are the dominant birds identified

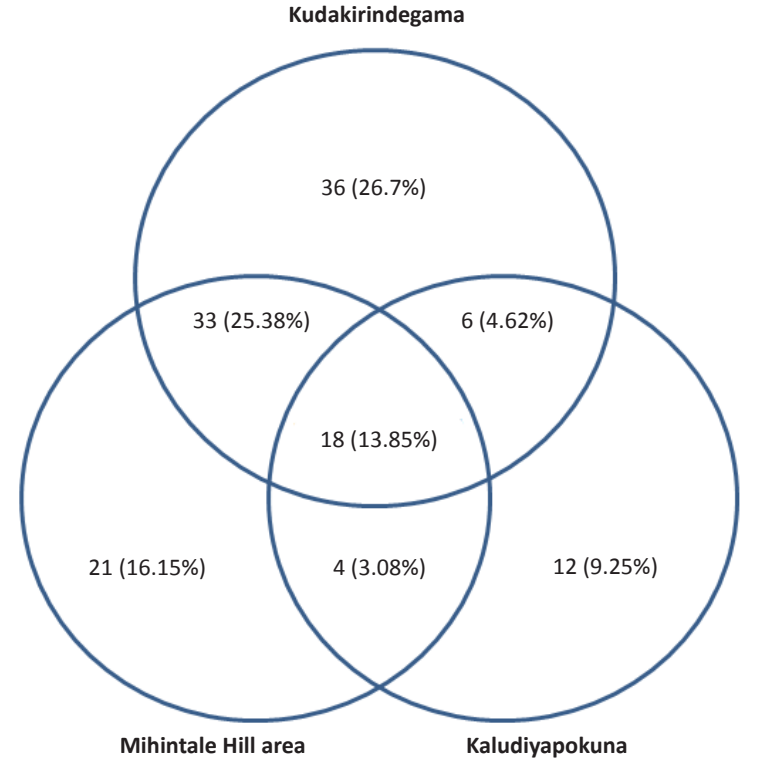

Figure 2. Species composition in different habitats within the Mihintale Sanctuary

at $\mathrm{MH}$.

\section{Discussion}

Out of the breeding resident species found in Sri Lanka, $48.9 \%$ were recorded within the sanctuary. So the species richness of avifauna of the whole sanctuary was quite high. However, the overall endemicity in the sanctuary was relatively low (15.4\%) compared to the protected areas in the wet zone (84.6\%). This is generally the expected result for dry zone habitats (Weerakoon \& Goonatilleke 2007). This may be due to the absence of suitable microclimatic conditions for these endemic birds. But the wet zone rainforests provide habitats for nearly all of the country's woody endemic plants and for about three-quarters of the endemic animals (Ministry of Forestry and Environment 1999). About $20 \%$ of the winter visitors were recorded during this survey.

Species richness and community structure of birds vary from region to region, as well as within a region, as abiotic and biotic factors vary from habitat to habitat (Johnsingh \& Joshua 1994). Of the three habitat types present in the sanctuary, highest species diversity was recorded in the $\mathrm{KK}\left(\mathrm{H}^{\prime}: 3.83\right)$, while the lowest species diversity was recorded in the KP forest ( $H^{\prime}$ : 3.32). According to the vegetation analysis, $45 \%$ of fruiting trees were present in KK while $25 \%$ of fruiting trees were recorded in KP forest. Hence, this variation in species richness may be due to the structural changes of vegetation and the availability of food resources. Bird 
Table 1. List of Avifauna in Mihintale Sanctuary

\begin{tabular}{|c|c|c|c|c|c|c|c|}
\hline Family & Common name & Scientific name & Status & КK & KP & MH & $\begin{array}{l}\text { Feeding } \\
\text { Guild }\end{array}$ \\
\hline \multirow{8}{*}{ Accipitridae } & Black-winged Kite & Elanus caeruleus & $\mathrm{BR}, \mathrm{C}$ & - & - & $x$ & $\mathrm{AC}$ \\
\hline & Brahminy Kite & Haliastur Indus & $B R, V C$ & $x$ & $x$ & $x$ & $A C$ \\
\hline & Changeable Hawk-Eagle & Spizaetus cirrhatus & $B R, C$ & $x$ & - & - & $A C$ \\
\hline & Crested Serpent Eagle & Spilornis cheela & $\mathrm{BR}, \mathrm{VC}$ & - & $x$ & $x$ & $\mathrm{AC}$ \\
\hline & Grey-headed FishEagle & Ichthyophaga ichthyaetus & $\mathrm{BR}, \mathrm{R}, \mathrm{NT}$ & $x$ & - & - & $\mathrm{AC}$ \\
\hline & Oriental Honey Buzzard & Pernis ptilorhyncus & $M, R$ & $x$ & $x$ & - & $A C$ \\
\hline & Shikra & Accipiter badius & $B R, V C$ & - & $x$ & $x$ & ATC \\
\hline & White-bellied Sea Eagle & Haliaeetus leucogaster & $\mathrm{BR}, \mathrm{C}$ & $x$ & $x$ & - & $\mathrm{AC}$ \\
\hline \multirow[t]{2}{*}{ Alaudidae } & Oriental Skylark & Alauda gulgula & $B R, C$ & - & - & $x$ & GI \\
\hline & Rufous-winged Bush-Lark & Mirafra assamica & $B R, V C$ & - & - & $x$ & GI \\
\hline \multirow{5}{*}{ Alcedinidae } & Black-backed Kingfisher & Cexy erithacus & $B R, R$ & - & - & $x$ & ATC \\
\hline & Common Kingfisher & Alcedo atthis & $B R, V C$ & $x$ & $x$ & $x$ & ATC \\
\hline & Stork-billed Kingfisher & Pelargopsis capensis & $\mathrm{BR}, \mathrm{C}$ & $x$ & - & - & ATC \\
\hline & White-throated Kingfisher & Halcyon smyrnensis & $B R, V C$ & $x$ & - & $x$ & ATC \\
\hline & Pied Kingfisher & Ceryle rudis & $\mathrm{BR}, \mathrm{C}$ & $x$ & 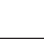 & & ATC \\
\hline \multirow{2}{*}{ Anatidae } & Cotton Pigmy-Goose & Nettapus coromandelianus & $\mathrm{BR}, \mathrm{C}$ & $x$ & - & - & $\mathrm{P}$ \\
\hline & Lesser Whistling Duck & Dendrocygna javanica & $B R, V C$ & $x$ & - & - & $\mathrm{P}$ \\
\hline \multirow{2}{*}{ Apodidae } & Asian Palm-Swift & Cypsiurus balasiensis & $\mathrm{BR}, \mathrm{C}$ & $x$ & - & $x$ & $\mathrm{Al}$ \\
\hline & Little Swift & Apus affinis & $B R, C$ & - & - & $x$ & $\mathrm{Al}$ \\
\hline \multirow{8}{*}{ Ardeidae } & Black-crowned Night-heron & Nycticorax nycticorax & $\mathrm{BR}, \mathrm{C}$ & $x$ & - & - & WC \\
\hline & Cattle Egret & Bubulcus ibis & $B R, V C$ & $x$ & - & $x$ & WC \\
\hline & Great Egret & Casmerodius albus & $\mathrm{BR}, \mathrm{C}$ & $x$ & - & - & WC \\
\hline & Grey Heron & Ardea cinerea & $\mathrm{BR}, \mathrm{C}$ & $x$ & - & - & WC \\
\hline & Indian Pond-heron & Ardeola grayii & $B R, V C$ & $x$ & - & $x$ & WC \\
\hline & Intermediate Egret & Mesophoyx intermedia & $B R, V C$ & $x$ & - & - & WC \\
\hline & Little Egret & Egretta garzetta & $B R, V C$ & $x$ & - & $x$ & WC \\
\hline & Purple Heron & Ardea purpurea & $B R, C$ & $x$ & - & - & WC \\
\hline Bucerotidae & Malabar Pied Hornbill & Anthracoceros coronatus & BR, C, NT & $x$ & - & $x$ & $\mathrm{AFI}$ \\
\hline Bucerotidae & Sri Lanka Grey Hornbill & Ocyceros gingalensis & $\mathrm{BR}, \mathrm{VC}, \mathrm{E}, \mathrm{NT}$ & $x$ & $x$ & $x$ & $\mathrm{AFI}$ \\
\hline \multirow{5}{*}{ Campephagidae } & Bar-winged Flycatcher & Hemipus pictatus & $\mathrm{BR}, \mathrm{C}$ & $x$ & - & - & $A B I$ \\
\hline & Black-headed Cuckooshrike & Coracina melanoptera & $B R, R$ & $x$ & - & - & FGI \\
\hline & Common Woodshrike & Tephrodornis pondicerianus & $B R, V C, P E$ & $x$ & - & $x$ & FGI \\
\hline & Large Cuckooshrike & Coracina macei & $\mathrm{BR}, \mathrm{C}$ & - & - & $x$ & $\mathrm{FGI}, \mathrm{F}, \mathrm{N}$ \\
\hline & Small Minivet & Pericrocotus cinnamomeus & $\mathrm{BR}, \mathrm{C}$ & $x$ & $x$ & $x$ & $\mathrm{FG}, \mathrm{I}$ \\
\hline \multirow{3}{*}{ Capitonidae } & Brown-headed Barbet & Megalaima zeylanica & $B R, V C$ & $x$ & $x$ & $x$ & AF \\
\hline & Coppersmith Barbet & Megalaima haemacephala & $\mathrm{BR}, \mathrm{C}$ & $x$ & - & $x$ & $\mathrm{AF}$ \\
\hline & Crimson-fronted Barbet & Megalaima rubricapilla & $B R, V C, P E$ & $x$ & $x$ & - & AF \\
\hline Caprimulgidae & Common Nightjar & Caprimulgus asiaticus & $\mathrm{BR}, \mathrm{C}$ & $x$ & - & - & $A B I$ \\
\hline Charadriidae & Red-wattled Lapwing & Vanellus indicus & $B R, V C$ & $x$ & - & - & $\mathrm{Gl}$ \\
\hline \multirow{3}{*}{ Ciconiidae } & Asian Open-Bill & Anastomus oscitans & $B R, C$ & $x$ & - & - & WC \\
\hline & Painted Stork & Mycteria leucocephala & BR, C, NT & $x$ & - & - & WC \\
\hline & Wooly-necked Stork & Ciconia episcopus & $B R, R$ & $x$ & - & - & WC \\
\hline \multirow{6}{*}{ Columbidae } & Emerald Dove & Chalcophaps indica & $\mathrm{BR}, \mathrm{C}$ & - & - & $x$ & GS \\
\hline & Green Imperial-pigeon & Ducula aenea & $B R, C$ & - & - & $x$ & $\mathrm{AF}$ \\
\hline & Orange-breasted Green-pigeon & Treron bicincta & $B R, C$ & $x$ & - & $x$ & AF \\
\hline & Pompadour Green-pigeon & Treron pompadora & $B R, C, P E$ & $x$ & - & $x$ & $\mathrm{AF}$ \\
\hline & Rock Pigeon & Columba livia & $B R, V C$ & - & - & $x$ & GS \\
\hline & Spotted Dove & Streptopelia chinensis & $\mathrm{BR}, \mathrm{VC}$ & $x$ & - & $x$ & GS \\
\hline Coraciidae & Indian Roller & Coracias bengalensis & $B R, C$ & - & - & $x$ & $A B I$ \\
\hline
\end{tabular}




\begin{tabular}{|c|c|c|c|c|c|c|c|}
\hline Family & Common name & Scientific name & Status & KK & KP & MH & $\begin{array}{l}\text { Feeding } \\
\text { Guild }\end{array}$ \\
\hline Corvidae & Large-billed Crow & Corvus macrorhynchos & $B R, V C$ & - & - & $\mathrm{x}$ & ATO \\
\hline \multirow{4}{*}{ Cuculidae } & Asian Koel & Eudynamys scolopacea & $\mathrm{BR}, \mathrm{VC}$ & $x$ & - & $x$ & $\mathrm{AF}$ \\
\hline & Blue-faced Malkoha & Phaenicophaeus viridirostris & BR, C & $x$ & $x$ & - & $\mathrm{AFI}$ \\
\hline & Greater Coucal & Centropus sinensis & $\mathrm{BR}, \mathrm{VC}$ & $\mathrm{x}$ & - & - & ATC \\
\hline & Grey-bellied Cuckoo & Cacomantis passerinus & $\mathrm{M}, \mathrm{C}$ & $x$ & - & $x$ & $\mathrm{ABI}$ \\
\hline \multirow[t]{2}{*}{ Dicaeidae } & Pale- billed Flower Pecker & Dicaeum erythrorhynchos & $\mathrm{BR}, \mathrm{VC}$ & $x$ & $x$ & $x$ & AF \\
\hline & Thick-billed Flower Pecker & Dicaeum agile & $B R, R$ & - & - & $x$ & $\mathrm{AF}$ \\
\hline Dicruridae & White-bellied Drongo & Dicrurus caerulescens & $B R, V C$ & $x$ & - & $x$ & $\mathrm{ABI}$ \\
\hline Hemiprocnidae & Crested Tree-Swift & Hemiprocne coronata & $\mathrm{BR}, \mathrm{C}$ & - & - & $x$ & $\mathrm{Al}$ \\
\hline \multirow{2}{*}{ Hirundinidae } & Barn Swallow & Hirundo rustica & $\mathrm{M}, \mathrm{VC}$ & $x$ & $x$ & $x$ & $\mathrm{Al}$ \\
\hline & Red-rumped Swallow & Hirundo daurica & $\mathrm{BR}, \mathrm{C}$ & $x$ & $x$ & - & $\mathrm{Al}$ \\
\hline \multirow{2}{*}{ Irenidae } & Blue-winged Leafbird & Chloropsis cochinchinensis & $\mathrm{BR}, \mathrm{C}$ & $x$ & - & $x$ & $\mathrm{FGI}, \mathrm{F}, \mathrm{N}$ \\
\hline & Common lora & Aegithina tiphia & $\mathrm{BR}, \mathrm{VC}$ & $x$ & $x$ & $x$ & $\mathrm{FG}, \mathrm{I}$ \\
\hline Jacanidae & Pheasant-tailed Jacana & Hydrophasianus chirurgus & $\mathrm{BR}, \mathrm{VC}$ & $\mathrm{x}$ & - & - & WC \\
\hline Laniidae & Brown Shrike & Lanius cristatus cristatus & $\mathrm{M}, \mathrm{C}$ & $\mathrm{x}$ & - & - & $\mathrm{ABI}$ \\
\hline Laridae & Whiskered Tern & Chlidonias hybridus & $\mathrm{M}, \mathrm{VC}$ & $x$ & - & - & $A C$ \\
\hline \multirow{3}{*}{ Meropidae } & Blue-tailed Bee-eater & Merops philippinus & $\mathrm{M}, \mathrm{VC}$ & $x$ & - & $x$ & $A B I$ \\
\hline & Chestnut-headed Bee-eater & Merops leschenaulti & $B R, C$ & $x$ & - & $x$ & Al \\
\hline & Little Green Bee-eater & Merops orientalis & $\mathrm{BR}, \mathrm{VC}$ & $x$ & - & $x$ & $\mathrm{ABI}$ \\
\hline \multirow{3}{*}{ Motacillidae } & Forest Wagtail & Dendronanthus indicus & $\mathrm{M}, \mathrm{C}$ & - & $x$ & $x$ & $\mathrm{TI}$ \\
\hline & Paddyfield Pipit & Anthus rufulus & $B R, V C$ & $x$ & - & $x$ & $\mathrm{Gl}$ \\
\hline & Richard's Pipit & Anthus richardi & $\mathrm{M}, \mathrm{C}$ & $x$ & - & $x$ & $\mathrm{Gl}$ \\
\hline \multirow{12}{*}{ Muscicapidae } & Ashy Prinia & Prinia socialis & BR, C & $x$ & - & $x$ & $\mathrm{Gl}$ \\
\hline & Asian Brown Flycatcher & Muscicapa dauurica & $\mathrm{M}, \mathrm{C}$ & $x$ & $x$ & $x$ & $\mathrm{ABI}$ \\
\hline & Asian Paradise Flycatcher-brown & Terpsiphone paradisi ceylonensis & BR, C & $x$ & $x$ & $x$ & $\mathrm{ABI}$ \\
\hline & Asian Paradise Flycatcher-white & Terpsiphone paradisi paradisi & $\mathrm{M}, \mathrm{C}$ & $x$ & $x$ & $x$ & $\mathrm{ABI}$ \\
\hline & Black-naped Monarch & Hypothymis azurea & $B R, C$ & $x$ & $x$ & - & $\mathrm{ABI}$ \\
\hline & Blyth's Reed Warbler & Acrocephalus dumetorum & $\mathrm{M}, \mathrm{C}$ & - & $x$ & - & FGI \\
\hline & Common Tailorbird & Orthotomus sutorius & $\mathrm{BR}, \mathrm{VC}$ & $x$ & - & $x$ & $F G, I$ \\
\hline & Dark-fronted Babbler & Rhopocichla atriceps & $\mathrm{BR}, \mathrm{C}$ & - & $x$ & - & $\mathrm{Tl}$ \\
\hline & Grey-breasted Prinia & Prinia hodgsonii & $B R, C$ & $x$ & - & $x$ & $\mathrm{Gl}$ \\
\hline & Indian Robin & Saxicoloides fulicata & $\mathrm{BR}, \mathrm{VC}$ & $x$ & - & $x$ & $\mathrm{TI}$ \\
\hline & Large-billed Leaf-Warbler & Phylloscopus magnirostris & $\mathrm{M}, \mathrm{C}$ & - & $x$ & - & FGI \\
\hline & Orange-headed Ground Thrush & Zoothera citrina & $M, V R$ & - & $x$ & - & $\mathrm{TI}$ \\
\hline \multirow{9}{*}{ Muscicapidae } & Oriental Magpie-Robin & Copsychus saularis & $\mathrm{BR}, \mathrm{VC}$ & $x$ & - & $x$ & $\mathrm{TI}$ \\
\hline & Plain Prinia & Prinia inornata & $B R, V C$ & $x$ & - & $x$ & $\mathrm{Gl}$ \\
\hline & Sri Lanka Brown-capped Babbler & Pellorneum fuscocapillum & $\mathrm{BR}, \mathrm{C}, \mathrm{E}$ & - & $x$ & - & $\mathrm{TI}$ \\
\hline & Tawny-bellied Babbler & Dumetia hyperythra & $B R, R$ & - & - & $x$ & FGI \\
\hline & Tickell's Blue Flycatcher & Cyornis tickelliae & $B R, C$ & - & $x$ & - & $\mathrm{TI}$ \\
\hline & White-rumped Shama & Copsychus malabaricus & $\mathrm{BR}, \mathrm{VC}$ & - & $x$ & - & FGI \\
\hline & Yellow-billed Babbler & Turdoides affinis & $\mathrm{BR}, \mathrm{VC}$ & $x$ & - & $x$ & TO \\
\hline & Yellow-eyed Babbler & Chrysomma sinense & $B R, R$ & - & $x$ & $x$ & $\mathrm{TI}$ \\
\hline & Yellowish Breasted Warbler & Phylloscopus nitidus & $\mathrm{M}, \mathrm{C}$ & - & $x$ & - & FGI \\
\hline \multirow{3}{*}{ Nectariniidae } & Long-billed Sunbird & Nectarinia lotenia & $B R, V C$ & $x$ & - & $x$ & AN \\
\hline & Purple Sunbird & Nectarinia asiatica & $B R, V C$ & $x$ & $x$ & $x$ & AN \\
\hline & Purple-rumped Sunbird & Nectarinia zeylonica & $\mathrm{BR}, \mathrm{VC}$ & $\mathrm{x}$ & $x$ & $x$ & AN \\
\hline Oriolidae & Black-hooded Oriole & Oriolus xanthornus & $\mathrm{BR}, \mathrm{VC}$ & $x$ & $x$ & $x$ & $\mathrm{FGI}, \mathrm{F}, \mathrm{N}$ \\
\hline Paridae & Great Tit & Parus major & $B R, C$ & - & - & $x$ & BGI \\
\hline
\end{tabular}




\begin{tabular}{|c|c|c|c|c|c|c|c|}
\hline Family & Common name & Scientific name & Status & KK & KP & MH & $\begin{array}{l}\text { Feeding } \\
\text { Guild }\end{array}$ \\
\hline Pelecanidae & Spot-billed Pelican & Pelecanus philippensis & $B R, V C, G T$ & $\mathrm{x}$ & - & - & $\mathrm{P}$ \\
\hline \multirow{3}{*}{ Phalacrocoracidae } & Indian Cormorant & Phalacrocorax fuscicollis & $B R, V C$ & $x$ & - & - & $\mathrm{P}$ \\
\hline & Little Cormorant & Phalacrocorax niger & $B R, V C$ & $x$ & - & - & $P$ \\
\hline & Oriental Darter & Anhinga melanogaster & $\mathrm{BR}, \mathrm{C}, \mathrm{NT}$ & $x$ & - & - & $P$ \\
\hline \multirow{2}{*}{ Phasianidae } & Indian Peafowl & Pavo cristatus & $\mathrm{BR}, \mathrm{C}$ & $x$ & $x$ & $x$ & TO \\
\hline & Sri Lanka Jungle Fowl & Gallus lafayetii & $\mathrm{BR}, \mathrm{C}, \mathrm{E}, \mathrm{NT}$ & $x$ & $x$ & $\mathrm{x}$ & TO \\
\hline \multirow{2}{*}{ Picidae } & Black-rumped Flameback & Dinopium benghalense & $\mathrm{BR}, \mathrm{C}$ & $x$ & - & - & BGI \\
\hline & Brown-capped-Woodpecker & Dendrocopos nanus & BR, C & - & - & $x$ & BGI \\
\hline Pittidae & Indian Pitta & Pitta brachyura & $\mathrm{M}, \mathrm{C}$ & - & $x$ & - & $\mathrm{TI}$ \\
\hline \multirow{5}{*}{ Ploceidae } & Baya Weaver & Ploceus philippinus & $B R, V C$ & $x$ & - & - & GS \\
\hline & Black-headed Munia & Lonchura malacca & $B R, V C$ & $x$ & - & - & GS \\
\hline & House Sparrow & Passer domesticus & $B R, V C$ & - & - & $x$ & GS \\
\hline & Scaly-breasted Munia & Lonchura punctulata & $B R, V C$ & $x$ & - & $x$ & GS \\
\hline & White-rumped Munia & Lonchura striata & $B R, V C$ & - & - & $x$ & GS \\
\hline Podargidae & Frogmouth & Batrachostomus monilieger & $B R, R$ & - & - & $x$ & $\mathrm{ABI}$ \\
\hline Podicipedidae & Little Grebe & Tachybaptus ruficollis & $\mathrm{BR}, \mathrm{C}$ & $x$ & - & - & $P$ \\
\hline \multirow{2}{*}{ Psittacidae } & Rose-ringed Parakeet & Psittacula kramerii & $B R, V C$ & $\mathrm{x}$ & $x$ & $x$ & $A F, N, G, S$ \\
\hline & Alexandra Parakeet & Psittacula eupatria & $\mathrm{BR}, \mathrm{C}$ & $x$ & $x$ & $x$ & $A F, N, G, S$ \\
\hline \multirow{4}{*}{ Pycnonotidae } & Black-crested Bulbul & Pycnonotus melanicterus & $B R, C, P E$ & - & $x$ & - & AFI \\
\hline & Red-vented Bulbul & Pycnonotus cafer & $B R, V C$ & $x$ & - & $x$ & $\mathrm{AFI}$ \\
\hline & White-browed Bulbul & Pycnonotus luteolus & $B R, V C$ & $\mathrm{x}$ & $x$ & $x$ & $\mathrm{AFI}$ \\
\hline & Yellow-browed Bulbul & lole indica & $B R, C$ & - & $x$ & - & $\mathrm{AFI}$ \\
\hline \multirow{3}{*}{ Rallidae } & Purple Swamp hen & Porphyrio porphyrio & $B R, V C$ & $x$ & - & - & WC \\
\hline & Salty-legged Crake & Rallina eurizonoides & $\mathrm{M}, \mathrm{C}$ & $x$ & - & - & WC \\
\hline & White- breasted Water Hen & Amaurornis phoenicurus & $B R, V C$ & $\mathrm{x}$ & - & $x$ & WC \\
\hline \multirow{2}{*}{ Scolopacidae } & Marsh Sandpiper & Tringa stagnatilis & $\mathrm{M}, \mathrm{VC}$ & $x$ & - & - & WC \\
\hline & Wood Sandpiper & Tringa glareola & $M, V C$ & $x$ & - & - & WC \\
\hline Strigidae & Brown Fish-Owl & Ketupa zeylonensis & $\mathrm{BR}, \mathrm{C}$ & - & $x$ & - & ATC \\
\hline Sturnidae & Common Mynah & Acridotheres tristis & $B R, V C$ & $\mathrm{x}$ & - & $x$ & ATO \\
\hline Threskiornithidae & Black-headed Ibis & Threskiornis melanocephalus & $B R, V C$ & $\mathrm{x}$ & - & - & WC \\
\hline Turnicidae & Barred Buttonquail & Turnix suscitator & $B R, C$ & - & - & $x$ & TO \\
\hline Zosteropidae & Oriental White-eye & Zosterops palpebrosa & $\mathrm{BR}, \mathrm{VC}$ & $x$ & - & $x$ & $\mathrm{FGI}, \mathrm{F}, \mathrm{N}$ \\
\hline 47 & & 130 species ( 4 endemics) & & 96 & 40 & 76 & \\
\hline
\end{tabular}

Feeding Guild: ABI - Arboreal Insectivore; AI - Areal Insectivore; FGI - Foliage Gleaning Insectivore; BGI - Bark-Gleaning Insectivore; TI - Terrestrial Insectivore; GI - grassland Insectivore; AC - Aerial Carnivore; ATC - Arboreal-Terrestrial Carnivore; WC - Wading Carnivore; AN - Arboreal Nectarivore; AF - Arboreal Frugivore; AFNGS - Arboreal Frugivore, Nectarivore, Granivore, Seed-eater; GS - Granivore, Seed-eater; AFI - Arboreal Frugivore, Insectivore; FGIFN - Foliage Gleaning Insctivore, Frugivore, Nectarivore; ATO - Arboreal-Terrestrial Omnivore; TO - Terrestrial Omnivore; P - Psicivore.

Status: BR - Breeding Resident; M - Migrant; VC - Very Common; C - Common; R - Rare; VR - Very Rare; E - Endemic; PE - Proposed Endemic; GT - Globally Threatened; NT- Nationally Threatened.

species distribution of an area may also depend on the insect and biomass of the area (Holmes \& Sherry 1997). These factors may change due to human influences, climatic and topographic conditions of the area.

Kudakirindegama area is composed of mainly aquatic habitat and is surrounded by many habitat types including grasslands, scrub forest, paddy fields etc. These habitat types provide suitable niches and food resources for many types of birds. Daniels (1989) found an increase in bird species diversity when forests are disturbed, as in disturbed forests fewer specialist species and more generalist species are seen, as was observed in $\mathrm{MH}$ where the species richness was high (76 bird species) compared to KP. This can be attributed to the fact that the primary habitat of KP area is rocky due to which availability of food resources is low although in certain areas there is presence of good amount of leaf litter. 
Table 2. Comparison of the total number of species and individuals of the avifauna at the three habitat types

\begin{tabular}{|c|c|c|}
\hline \multicolumn{2}{|c|}{ Sites } & $\mathrm{P}$ value \\
\hline \multirow{4}{*}{ Total number of Species } & $\mathrm{K}{ }^{*} \mathrm{KP} * \mathrm{MH}$ & $\mathrm{P}=0.000^{*}$ \\
\cline { 2 - 3 } & $\mathrm{KK} * \mathrm{MH}$ & $\mathrm{P}=0.065$ \\
\cline { 2 - 3 } & $\mathrm{KK} * \mathrm{KP}$ & $\mathrm{P}=0.000^{*}$ \\
\cline { 2 - 3 } & $\mathrm{KP} * \mathrm{MH}$ & $\mathrm{P}=0.001^{*}$ \\
\hline \multirow{4}{*}{ Total number of Individuals } & $\mathrm{KK}$ * KP*MH & $\mathrm{P}=0.000^{*}$ \\
\cline { 2 - 3 } & $\mathrm{KK} * \mathrm{MH}$ & $\mathrm{P}=0.078$ \\
\cline { 2 - 3 } & $\mathrm{KK} * \mathrm{KP}$ & $\mathrm{P}=0.001^{*}$ \\
\cline { 2 - 3 } & $\mathrm{KP} * \mathrm{MH}$ & $\mathrm{P}=0.002^{*}$ \\
\hline
\end{tabular}

KK - Kudakirindegama; KP - Kaludiyapokuna; MH - Mihintale Hill area

Since understory birds are the most sensitive to disturbances in forest structure, they ought to be reliable indicators of forest regeneration (Wong 1985). Among tropical forest birds, understory insectivores are particularly sensitive to habitat disturbance and fragmentation (Sekercioglu et al. 2002). So the bird species like Zoothera citrina, Rhopocichla atriceps, Acrocephalus dumetorum, Pellorneum fuscocapillum, Cyornis tickelliae, Copsychus malabaricus etc, which are found only in KP forested area can be considered as indicator species for forest fragmentation and disturbance.

Bird species like Columba livia, Corvus macrorhynchos, Hemiprocne coronata, Parus major, Passer domesticus, Lonchura striata are confined to $\mathrm{MH}$ disturbed area and since they live in disturbed habitats are able to tolerate all the disturbances and pollution caused to the habitat. Fragmentation of large tracks of natural ecosystems in to small patches is one of the major impediments to long term conservation of biodiversity. Improper land use planning is the primary cause of fragmentation. Fragmentation also results in the reduction of species richness as species richness is dependent on the extent of the habitat (Rodrigo 2007). The forest of Kaludiyapokuna is fragmented in to small patches by the land use practices of nearby villagers. Which could be a reason for lowest species diversity $\left(H^{\prime}: 3.32\right)$ in $K P$, and also bird species like Zoothera citrina, Rhopocichla atriceps, Pellorneum fuscocapillum were observed in low numbers in this habitat due to the low extent of the forest habitat.

Lack of demarcation of boundaries is one of the major reasons for the increased human activities within the sanctuary. Therefore proper boundaries should be established and manned to minimize this human encroachment. Although the sanctuary harbours large

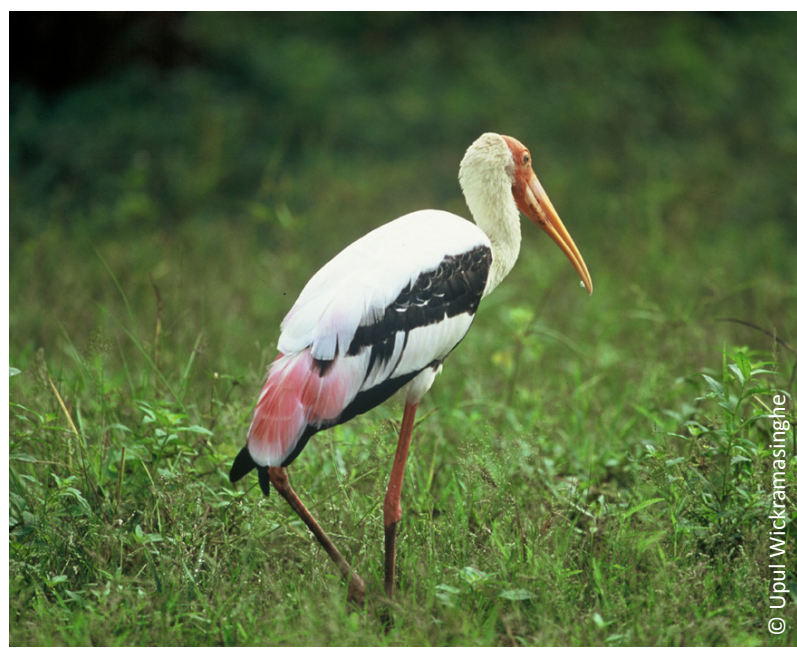

Image 1. Mycteria leucocephala

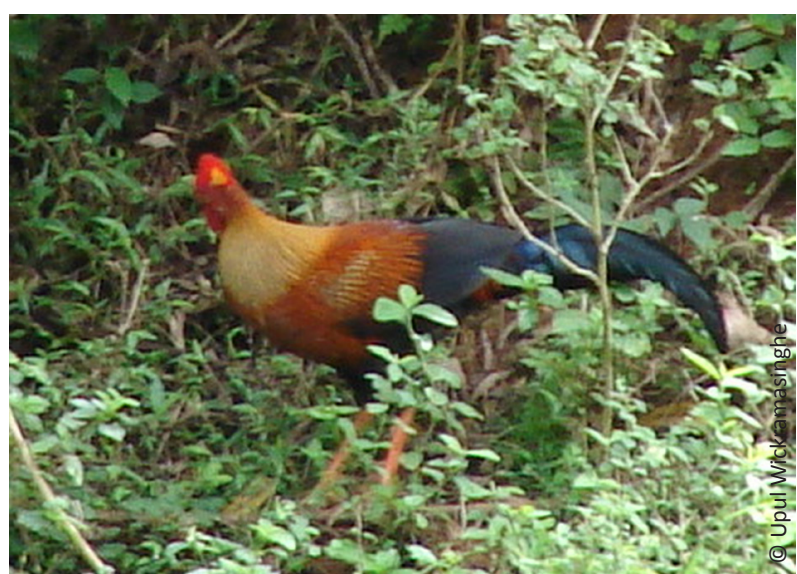

Image 2. Gallus Iafayetii

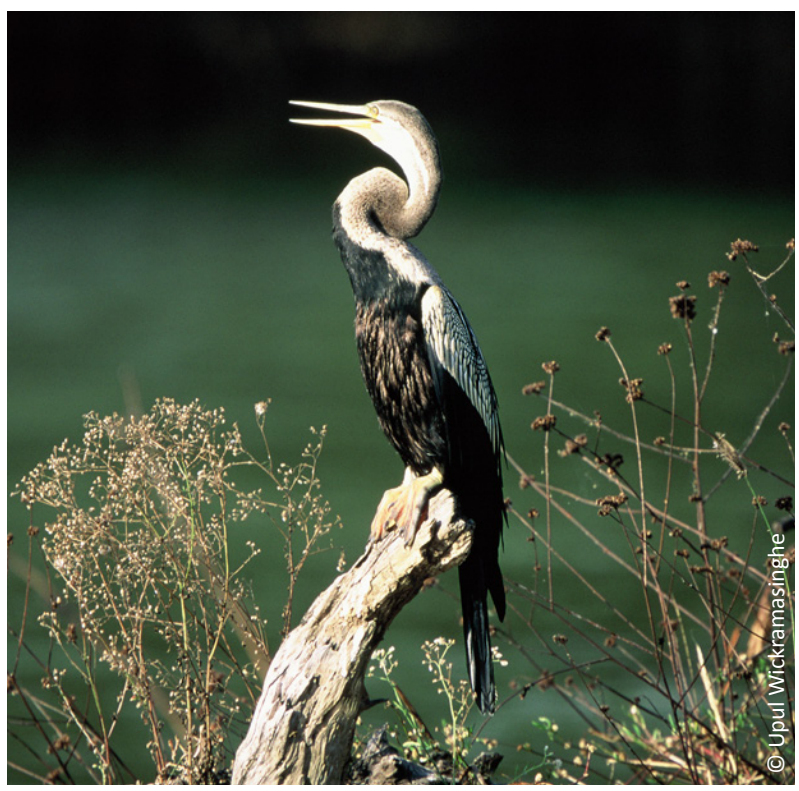

Image 3. Anhinga melanogaster 


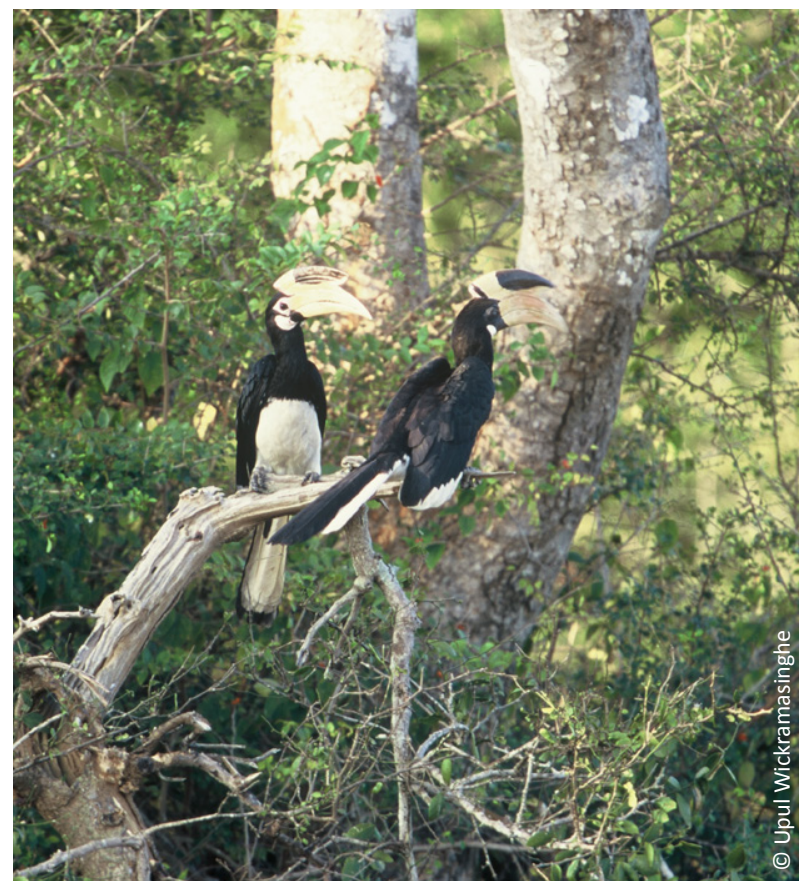

Image 4. Anthracoceros coronatus

number of birds, a proper conservation action plan is not in place and as this area is also an archeological site it is frequently subjected to the mining or excavation. There is no adverse effect to the habitat by tourists who visit the sanctuary in general. But the pressure is intense on the days of the main festival during the month of June.

\section{Conclusions}

The Brown-capped Babbler (Pellorneum fuscocapillum), is endemic to Sri Lanka, and birds such as this species need to be conserved by taking steps to reduce habitat fragmentation. Strategies should be developed to conserve the bird diversity that exists in the forest patches and further research is needed to help conserve the forest fragments.

\section{References}

Bibby, C.J., N.D. Burgess \& D.A. Hill (1993). Bird Census Techniques. Academic Press Limited, London, England, 302pp.

BirdLife International (2008). Birds of Sri Lanka <http://www. ibasrilanka.net/birds.Ik/threatened.asp>. Downloaded on 12 October 2008.

Daniels, R.J.R. (1989). A conservation strategy for the birds of the Uttara Kannada District. MPhil Thesis. Indian Institute of Science, Bangalore.

Department of Government Printing (1938). Sri Lanka Gazette Notification 1938-05-27. No. 8370: 5.

Divisional Secretary (2007). Sampath Pathikada. Mihintale, pp.12-35.

Harrison, J. (1999). A Field Guide to The Birds of Sri Lanka. Oxford University Press, New York, 434pp.

Holmes, R.T. \& T.W. Sherry (1997). Ecological factors influencing biodiversity in northern hardwoods ecosystems: contributions of long-term studies of bird populations at Hubbard Brook, 13pp.

Johnsingh, A.J.T. \& J. Joshua (1994). Avifauna in three vegetation types on Mundanthurai Plateau, South India. Journal of Tropical Ecology 10: 323-335.

Kotagama, S. \& P. Fernando (1994). A Field Guide to The Birds of Sri Lanka. The Wildlife Heritage Trust of Sri Lanka, Sri Lanka, 257pp.

Kotagama, S. \& A. Wijayasingha (1998). Sirilaka Kurullo. The Wildlife Heritage Trust of Sri Lanka, Sri Lanka.

Kotagama, S.W. \& G. Ratnavira (2010). An Illustrated Guide to The Birds of Sri Lanka. Field Ornithological Group of Sri Lanka, Sri Lanka. 357

Kotagama, S.W., R.I. de Silva, A.S. Wijayasingha \& V. Abeygunawardena (2006). Avifaunal list of Sri Lanka, pp. 164-203. In: Bambaradeniya, C.N.B. (eds.). Fauna of Sri Lanka: Status of Taxonomy, Research and Conservation. The World Conservation Union, Colombo, Sri Lanka and Government of Sri Lanka.

Ministry of Forestry and Environment (1999). Biodiversity Conservation in Sri Lanka: A Framework for Action, 5-13pp.

Rodrigo, M. (2007). Field ornithology group of Sri Lanka. Department of Zoology, University of Colombo. Siyoth 2(1): 39-42.

Sekercioglu, C.H., P.R. Ehrlich, G.C. Daily, D. Aygen, D. Goehring \& R.F. Sandi (2002). Disappearance of insectivorous birds from tropical forest fragments. Proceedings of the National Academy of Sciences 99(1): 263-267

Weerakoon, D.K. \& W.L.D.P.T.S.D.A Goonatilleke (2007). Diversity of avifauna in the Wilpattu National Park. Siyoth 6(2): 11-18.

Wimalathilake, E.M.A.S.K. (2005). Human impacts and its effect on avifauna at Mihintale sanctuary. Post Graduate Diploma Thesis. Post Graduate Diploma in Environmental Sciences, Open University of Sri Lanka, Nawala, 267pp.

Wong, M. (1985). Understory birds as indicators of regeneration in a patch of selectively logged West Malaysian rainforest. ICBP Technical Publication 4: 249-263. 